\title{
Diagnostic Accuracy of Digital Mammography in the Detection of Breast Cancer
}

\author{
Muhammad Zeeshan ${ }^{1}$, Basit Salam ${ }^{2}$, Qazi Saad B. Khalid ${ }^{3}$, Shahbaz Alam ${ }^{1}$, Raza Sayani ${ }^{4}$ \\ 1. Radiology, Royal Blackburn Hospital / East Lancashire Hospital Trust (ELHT), Manchester, GBR 2. \\ Radiology, Aga Khan University, Karachi, PAK 3. Radiology, Dallah Hospital, Dallah, SAU 4. Department \\ of Radiology, The Aga Khan University, Karachi, PAK
}

$\square$ Corresponding author: Raza Sayani, sayani_raza@yahoo.com

Disclosures can be found in Additional Information at the end of the article

\section{Abstract}

\section{Introduction}

Breast cancer has a high prevalence in the community and places very high demands on resources. Digital mammography provides a good quality image with reduced radiation dose and can detect breast carcinoma in its earlier stages, resulting in good prognosis and improved patient survival.

\section{Objective}

To calculate the diagnostic accuracy of digital mammography in the detection of breast cancer, using histopathology as a gold standard in women aged over 30 years, who are undergoing mammography for screening and diagnostic purposes.

\section{Materials and methods}

This was a cross-sectional analytical study, conducted in the department of radiology, for a total duration of 10 months. A total of 122 patients of age above 30 years, referred for digital mammography for the evaluation of different symptoms related to breast diseases, followed by biopsy/surgery and histopathology, were included in the study.

\section{Result}

Our data confirmed that digital mammography is a highly accurate tool for breast cancer detection having a sensitivity of $97 \%$, a specificity of $64.5 \%$, a positive predictive value of $89 \%$, and a negative predictive value of $90.9 \%$, with a diagnostic accuracy of $89.3 \%$.

Received 03/28/2018

Review began 03/30/2018 Review ended 04/05/2018 Published 04/08/2018

(C) Copyright 2018

Zeeshan et al. This is an open access article distributed under the terms of the Creative Commons Attribution License CC-BY 3.0., which permits unrestricted use, distribution, and reproduction in any medium, provided the original author and source are credited.

\section{Conclusion}

Considering our results, we recommend that digital mammography should replace screen-film mammography as a basic tool to detect breast cancer for both screening and diagnostic purposes.

Categories: Pathology, Radiology, Quality Improvement Keywords: breast cancer, digital mammography, spiculated density, pleomorphic microcalcifications, architectural distortion, skin thickening, nipple retraction 


\section{Introduction}

Breast cancer is the most common cancer in females in the United States and throughout the world. It is responsible for $21 \%$ of new cancer cases worldwide [1]. It is also prevalent among women in Pakistan (accounting for one-third of the cancers in females) [2]. The five-year survival of stage IV breast cancer is $10 \%$. However, earlier detection and treatment can improve five-year survival to $85 \%$ [1].

A study conducted by Karin Flobbe et al. in the Netherlands has estimated the prevalence of breast cancer to be $6.3 \%$, which is consistent with the results of other studies [3]. Breast cancer detection via mammography has been recommended traditionally and the majority of women older than 40 years in the United States participate [4]. Recent research conducted on 210,000 women in Sweden has shown that in women aged $40-49$ years, there was a significant $48 \%$ reduction in breast cancer mortality in those exposed to screening, whereas there was none in those unscreened [5].

Both screen-film mammography and digital mammography use x-rays to obtain images. With screen-film mammography, the image is captured on film; with digital mammography, the image is captured digitally [6]. Digital mammography is developed to address the limitations of film mammography and separates image acquisition and display, allowing the optimization of both [7].

In many recent studies, digital mammography was significantly better than conventional film mammography at detecting breast cancer in young women, premenopausal and perimenopausal women, and women with dense breasts [8]. The visibility of a subtle mass or cluster of microcalcifications present in the image can be increased if image contrast is adjusted [9]. Approximately 25-43\% of non-palpable cancers are detected on mammography because of microcalcifications [10].

Digital mammography also offers other benefits over film mammography in the form of easier access to images and computer-assisted diagnosis; improved means of transmission, retrieval, and storage of images; and the use of a lower average dose of radiation without compromising diagnostic accuracy [7].

Pisano et al., in one of their studies, calculated the sensitivity and specificity of digital mammography to be $85 \%$ and $90 \%$, respectively, in females aged less than 50 years and pre- or perimenopausal women with non-dense breasts [11].

The aim of this study is to calculate the diagnostic accuracy of digital mammography in detecting breast cancer early, using histopathology as the gold standard in women aged over 30 years who are undergoing mammography for screening and diagnostic purposes.

As the early detection of breast cancer increases the chances of cure and subsequently improves quality of life, this study will provide a landmark for other institutions to acquire and use digital mammography equipment for screening purposes.

\section{Materials And Methods}

This was a cross-sectional analytical study conducted in the department of radiology, for a total duration of 10 months. The sampling technique was non-probability, purposive. The sample size was calculated using sample size determination in the health studies manual by WHO. The reported sensitivity of a digital mammogram to detect breast cancer is $85 \%$ and the reported specificity is $90 \%$ [11]. The prevalence of breast cancer is $20 \%$ (one in five female patients). The desired precision is $12 \%$, so 121 patients were the required sample. We included 122 patients in 
the study.

All female patients above 30 years of age referred to the radiology department for digital mammography followed by biopsy were included. Patients who were already diagnosed with breast cancer and underwent digital mammography for follow-up and those patients who had inconclusive mammograms due to increased breast density and needed an ultrasound for further evaluation were excluded. Similarly, patients whose histopathology results were not available or they did not undergo biopsy/surgery were also excluded.

Informed consent was taken for the examination and inclusion in the study. After explaining the procedure, a brief history was taken regarding the patient's signs and symptoms, their duration, and any family history of breast cancer. Mammograms were performed on Mammomat Nova 3000 (Siemens AG, Munich, Germany) at 26-30 Kvp and mAs were set on automatic exposure control. Automatic exposure control is a default setting in digital mammographic equipment to control the exposure and is calibrated by an experienced physicist having a minimum of five years' experience.

Images were acquired in the mediolateral oblique and craniocaudal projections. When needed, additional views, such as exaggerated view, axillary view, magnified view, and cone compression view, were taken. The digital mammograms were analyzed by a post-graduate consultant radiologist with minimum five years' experience, while the histopathology results were provided by a post-graduate consultant pathologist with minimum five years' experience, which served as the gold standard. The findings of digital mammography, histopathology, and demographics were entered in the proforma by the researcher.

The patient's data were collected using a standard proforma. Data entry and analysis were done through IBM SPSS for Windows, version 19.0 (IBM Corp, Armonk, NY). Descriptive statistics were calculated. Mean and standard deviation were calculated for the age of the patient. Frequencies and percentages were calculated for the site of the lesion in the breast, any family history of breast cancer, the associated pleomorphic microcalcifications, architectural distortion, skin thickening and nipple retraction, and presence or absence of axillary lymph nodes.

Confounding variables, like age, family history of breast cancer, and use of hormone replacement therapy (HRT), were dealt with in data analysis and stratification was done to see the effect of these on the outcome variable.

A 2x2 table was used to calculate sensitivity, specificity, positive predictive value, negative predictive value, and diagnostic accuracy. Sensitivity, specificity, positive and negative predictive values, and diagnostic accuracy of digital mammography in the detection of breast cancer were calculated against histopathology as the gold standard, as defined in the operational definition.

\section{Results}

A total of 122 patients were included in our study according to inclusion and exclusion criteria, with a mean age of 50.79 years (SD +/- 12.06). Among these patients, spiculated density in the mammograms was present in 88 patients (72.1\%) (Figure 1). 


\section{Cureus}

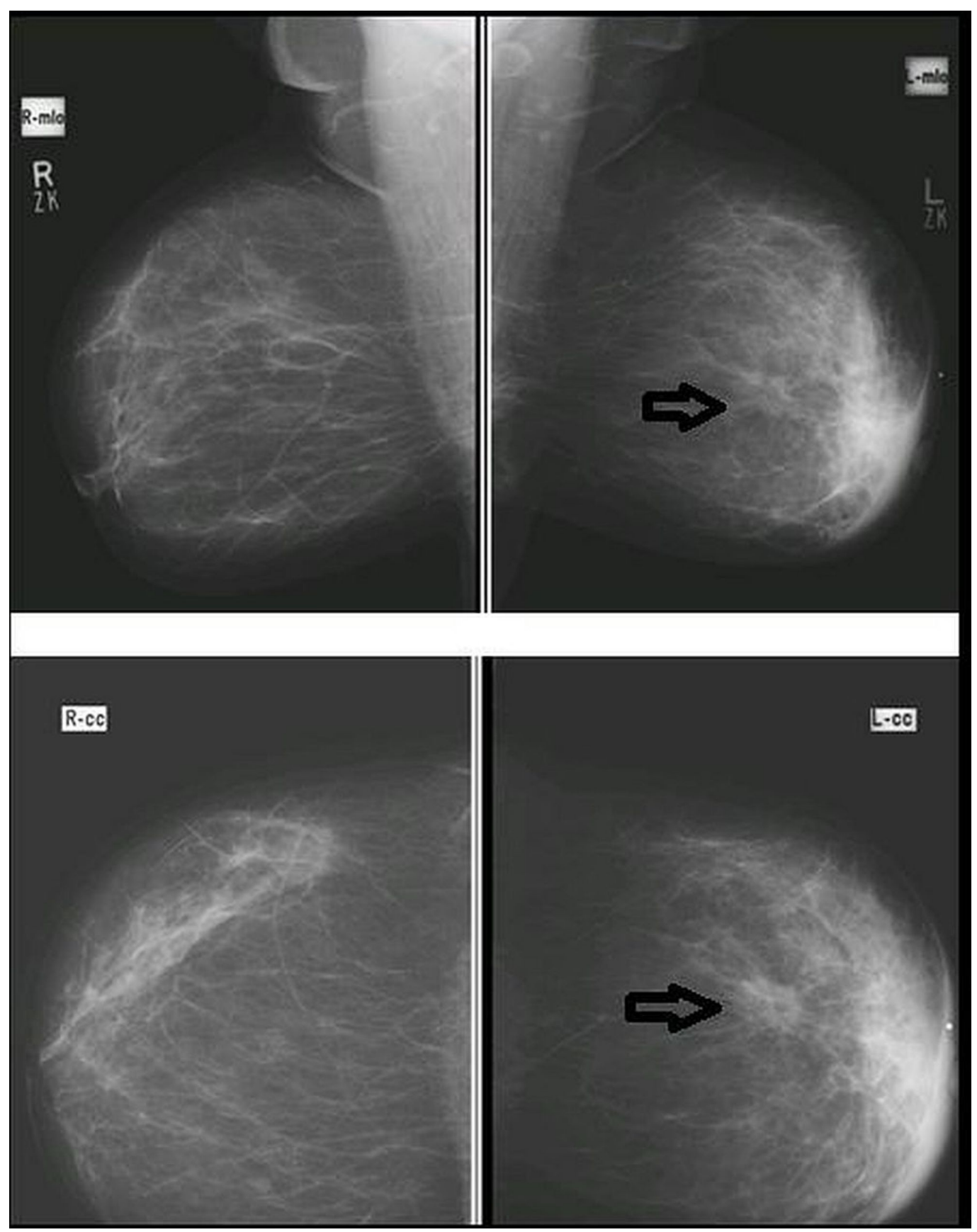

FIGURE 1: Mediolateral-oblique (MLO) and cranial-caudal (CC) views in a patient with spiculated density (arrow), architectural distortion, skin thickening, and nipple retraction of the left breast, proven to be breast carcinoma on histopathology (true positive).

The sizes of the spiculated density were grouped into either less than two $\mathrm{cm}$ or greater than two $\mathrm{cm}$. No spiculated lesion was found in $27.9 \%$ of the patients. Lesions that were $<2 \mathrm{~cm}$ in size were $18.9 \%$ and those $>2 \mathrm{~cm}$ were $53.3 \%$. The most common observed site of the lesion was the right upper-outer quadrant 40 (32.8\%), followed by the left upper-outer quadrant 34 (27.9\%). The pleomorphic microcalcification seen was present in 34 patients (28\%) and was absent in 88 (72\%). Architectural distortion was seen in 45 patients (36.9\%) and was absent in 


\section{Cureus}

77 patients (63.1\%). Skin features, such as skin thickening and nipple retraction, were present in $22(18 \%)$ and $10(8.2 \%)$ patients, respectively. Axillary lymph nodes were present in 106 patients (86.9\%). Similarly, breast cancer in mammograms was present in 100 patients (82\%) (Figure 2).
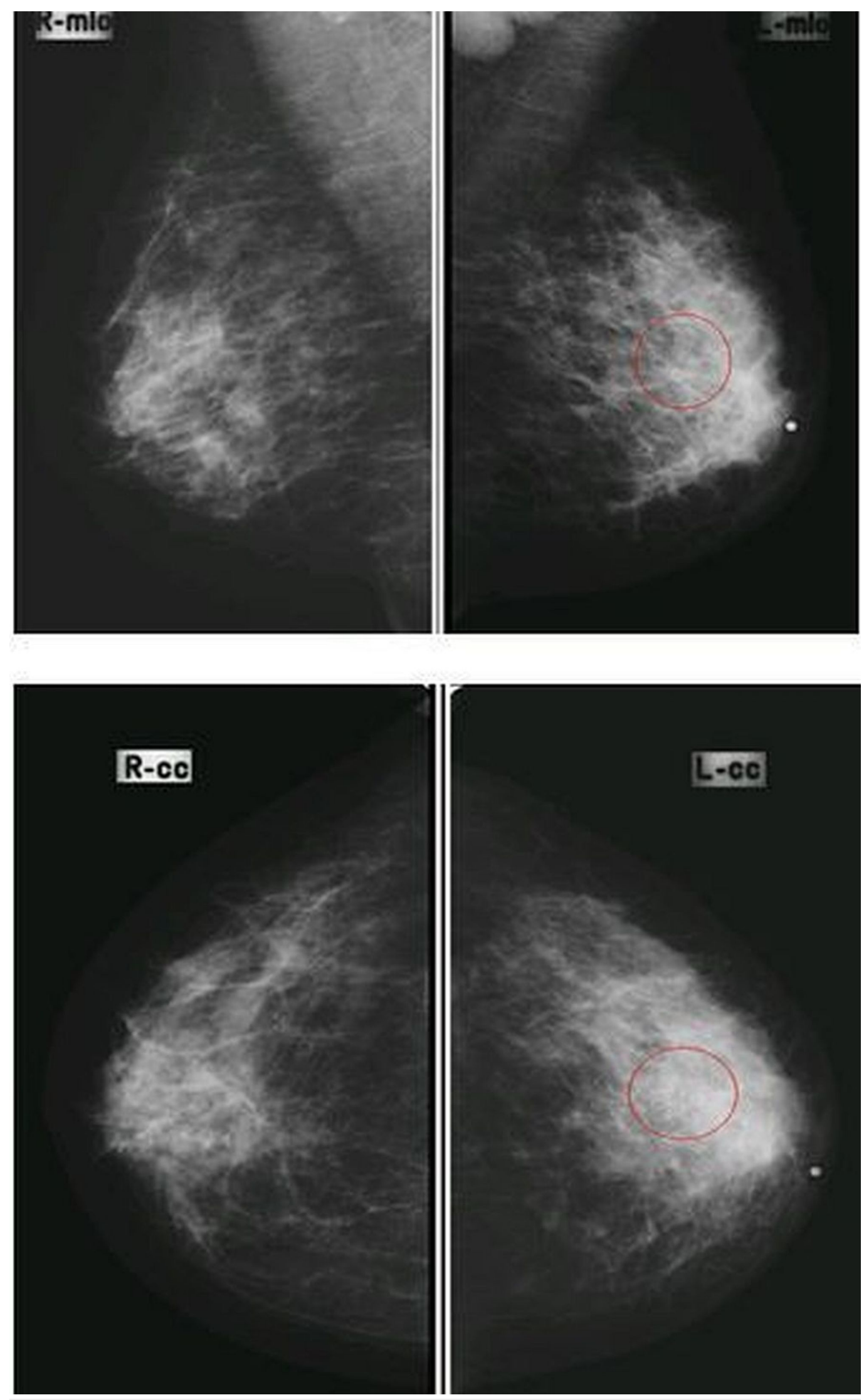
FIGURE 2: Mediolateral-oblique (MLO) and cranial-caudal (CC) views in a patient having pleomorphic microcalcifications in the upper-outer quadrant of the left breast with enlarged dense axillary lymph nodes, proven to be breast carcinoma on histopathology (true positive).

The frequencies of different histopathological features, such as atypical cells, increased mitotic activity, and hyperchromasia were present in $74.6 \%$ of the patients and were absent in $25.4 \%$.

A diagnosis of breast cancer on histopathology was seen in 91 patients (74.6\%).

Diagnostic accuracy was calculated as follows:

Sensitivity $=$ True Positive (89)/True Positive (89) + False Negative (2) x $100=97 \%$

Specificity $=$ True Negative $(20) /$ False Positive $(11)+$ True Negative $(20) \times 100=64.5 \%$

Positive predictive value $=$ True Positive (89)/True Positive (89) + False Positive (11)x100 = 89\%

Negative predictive value $=$ True Negative $(20) /$ False Negative $(2)+$ True Negative $(20) \times 100=$ $90.9 \%$

Diagnostic accuracy $=$ True Positive (89) + True Negative (20)/True Positive (89) + False Positive $(11)+$ False Negative (2) + True Negative (20) x 100=89.3\%

A family history of breast cancer was present in 10 patients (8.1\%), out of which eight had breast carcinoma (6.5\%) and two did not have breast carcinoma (1.6\%). The use of hormone replacement therapy (HRT) was present in six patients (4.9\%), out of which four had breast carcinoma (3.2\%) and two did not have breast carcinoma (1.6\%).

\section{Discussion}

Breast cancer has a high prevalence in the community and places very high demands on resources. Historically, ongoing efforts are made to detect it as early as possible by using different clinical and imaging techniques. Self breast examination and routine clinical checkups are clinical ways of detecting breast cancer early. However, due to the occult nature of most of the early breast cancers, imaging has a vital role to play.

The evolution of mammography dates back to 1913, when surgeon A Salomon conducted a study on the correlation between x-ray findings and histopathology in 3000 mastectomy cases [12]. Conventional mammograms have been replaced by digital mammography, which provides good quality images using reduced radiation doses and can detect breast carcinoma in its earlier stages, resulting in a good prognosis and improved patient survival.

In our study, a total of 122 patients were included according to the inclusion and exclusion criteria. They had a mean age of 50.79 years (SD +/- 12.06) with a minimum age of 31 years and a maximum age of 77 years. Out of the 122 patients, the highest number of patients belonged to the age group between 50 and 60 years (38 patients). The second-highest number of patients belonged to the age group 31-40 years (29 patients). The third-highest group was 40-50 years 
(25 patients). The patients who had breast cancer on a mammogram, which was later confirmed on histopathology, were of ages ranging from 43-60 years. Iqbal et al., in their study on the survival of patients having locally advanced breast cancer performed in a teaching hospital of Lahore, Pakistan, described the median age of breast cancer patients as 45 years [13]. They also described that most of the women were pre-menopausal, with a receptor-negative disease. Similar statistics were described in other studies performed in developing countries [14].

We calculated the diagnostic accuracy of digital mammography in detecting breast cancer to be $89.3 \%$, with a sensitivity of $97 \%$, a specificity of $64.5 \%$, a positive predictive value of $89 \%$, and a negative predictive value of $90.9 \%$. Pisano et al., in 2008 , performed a study and calculated the sensitivity and specificity of digital mammography in different age groups after considering their pre-, peri- or post-menopausal status and breast density [11]. In patients aged $<50$ years with a non-dense breast, the sensitivity was $85 \%$ with a specificity of $90 \%$ while in patients aged > 50 years with non-dense breast, the sensitivity was $66 \%$ and specificity was $93 \%$ [11]. In our study, the results were similar with a little less specificity, as the number of false positives was high due to a greater degree of suspicion considering the increasing prevalence of breast cancer in our country.

In this study, spiculated density was present in 34 patients (28\%) and 27 patients were confirmed to have breast carcinoma on histopathology (27/34, 79.4\%). The remaining seven patients having spiculated density on a digital mammogram were proven to have phyllodes tumor (4/7), surgical scarring (2/7), and benign sclerosing lesion (1/7). Most of the patients having spiculated density on digital mammograms had a palpable abnormality, while the rest were detected incidentally. All patients having a palpable abnormality usually had spiculated densities measuring more than two $\mathrm{cm}$ (62\% cases). Ciato et al. calculated the frequency of spiculated density in his study as 40\% (159/397 patients) [15].

The frequency of spiculated density in our study was a little higher due to the late presentation of the patients, as occurs in our country due to less awareness and low socioeconomic status.

The highest number of lesions was located in the right upper outer quadrant (40 cases; 32.8\%) with the second-most common location being the left upper-outer quadrant (34 cases; 27.9\%), emphasizing the fact of the occurrence of breast cancer commonly in the upper-outer quadrant. This is consistent with the literature results, which also showed that lesions are more common in the upper-outer quadrants. Naeem et al. shared their experience at Lady Reading Hospital, where they included 46 patients having breast cancer; out of them, 26 had lesions in the upperouter quadrants (26/46, 56.5\%) [16].

Pleomorphic microcalcifications were present in 34 cases (28\%), out of which 29 (29/34; 85.2\%) cases were diagnosed as having breast carcinoma on histopathology. In the past, the literature showed that pleomorphic microcalcification has a positive predictive value of $42 \%$ for the detection of malignancy [17]. Our study results are showing a higher positive predictive value of pleomorphic microcalcifications due to the use of digital mammography instead of screen-film mammography, as the former has better contrast resolution and the ability to magnify images digitally.

The indirect features suggesting breast cancer on digital mammograms are architectural distortion, skin thickening, and nipple retraction; these were present in 45 patients (36.9\%), 22 (18\%), and $10(8.2 \%)$ of patients, respectively.

Overall, our results are in concordance with studies done in the past regarding the frequencies of different mammographic signs in the detection of breast carcinoma. Goedde et al., in his study, found that spiculated density was present in $44 \%$ cases, followed by clustered 
microcalcifications in $25 \%$, mass in $22 \%$, and asymmetric density in $14 \%$ of the cases [18].

The presence of axillary lymph nodes on a digital mammogram is not an indicator of breast carcinoma, as it can be seen in normal patients as well as patients having a different diagnosis than breast cancer. Therefore, it is not a criterion for diagnosing breast cancer on mammograms. We calculated the frequency of axillary lymph nodes presence in digital mammograms, which was $87 \%$ in 122 patients.

Family history is an important risk factor in the development of breast cancer, especially at an early age. We evaluated the frequency of positive family history in patients having breast cancer, and out of 83 patients, eight had a positive family history (8/83; 9.63\%). Similar results have been quoted by past studies, including a study conducted by Chauhan et al., who in a hospital-based descriptive study, calculated the frequency of family history in breast cancer to be $8 \%$ [19]. Mai et al. calculated the sensitivity, specificity, and negative and positive predictive values of positive family history for different body cancers in a population-based study. He found that in breast cancer, family history has a sensitivity, specificity, positive predictive value, and negative predictive value of $61.1 \% .95 \%, 61.3 \%$, and $95 \%$, respectively [20].

Hormone replacement therapy (HRT) as a cause of breast cancer is a topic of great debate. It has been postulated that it is associated with a higher incidence of breast cancer in postmenopausal women. This has been based on evidence from three studies, the Collaborative Reanalysis (CR), the Women's Health Initiative (WHI), and the Million Women Study (MWS), which established the casual relationship. We calculated the frequency of HRT use in patients with breast cancer, which was found to be four out of 87 patients $(4 / 87 ; 4.59 \%)$. Czernichow et al. performed a retrospective analysis on the database of Institute Curie where it was found that 1482 patients used HRT for more than six months out of 6737 patients diagnosed with breast cancer (1482/6737; 21.9\%) [21]. This difference is because, in our country, hormone replacement therapy is not very popular and not prescribed by physicians on a regular basis.

Considering our study results, we can safely advocate that digital mammography can replace screen-film mammography in our country as well. The most important advantage of using digital mammography is a reduction in the radiation dosage that used to occur with the screenfilm technique because of film retakes. Akhtar et al. conducted a study in our department in 2008 comparing the retake rates in conventional versus digital radiography. He concluded that digital radiography had a significantly lower number of retakes (1\%) in comparison to the retakes performed in conventional radiography (5.5\%) [22].

This study also validates the need for nationwide breast cancer screening programs starting from the age of 40 years and above, as all the patients having breast cancer in our study fall in the range of 44-60 years.

There are a few disadvantages of digital mammography over screen-film mammography. First, the initial cost of installing the system is high as compared to screen-film mammography, but in the long term, it is cost-effective. Second, because digital mammographic equipment has been launched by different manufacturers all around the world, and they are based on different kinds of technologies, it is hard to recommend which one type of digital mammographic equipment should be used. As comparative data is not available yet, it is impossible to recommend, at this point in time, the best digital mammography equipment to be used for the best results [23].

In this study, we also recognized some limitations. First, we excluded those patients having dense breast parenchyma from digital mammograms, as it reduces its sensitivity of detection by digital mammography. But, we kept the heterogeneously dense breast parenchyma, which also 
reduces the sensitivity of digital mammograms. This might be the fact that may have resulted in false negative cases. Second, we did not stratify the results according to breast density, as many studies in the past have shown different diagnostic accuracies for different degrees of breast density and patterns of parenchyma [11]. Third, we did not compare the diagnostic accuracies of conventional screen-film mammography with those of digital mammography, as has been done in the literature [7]. The fourth limitation is that we did not calculate the interobserver variability, as the digital mammograms were interpreted by a single radiologist.

\section{Conclusions}

To summarize, our data confirmed that digital mammography is a highly accurate tool for breast cancer detection, having a sensitivity of $97 \%$, a specificity of $64.5 \%$, a positive predictive value of $89 \%$, and a negative predictive value of $90.9 \%$, with a diagnostic accuracy of $89.3 \%$. Digital mammography enjoys a few advantages over the film-screen conventional mammography and is replacing the older technique all over the world. However, special attention should be given while selecting equipment, as not much data is available for reference.

\section{Additional Information Disclosures}

Human subjects: Consent was obtained by all participants in this study. Animal subjects: All authors have confirmed that this study did not involve animal subjects or tissue. Conflicts of interest: In compliance with the ICMJE uniform disclosure form, all authors declare the following: Payment/services info: All authors have declared that no financial support was received from any organization for the submitted work. Financial relationships: All authors have declared that they have no financial relationships at present or within the previous three years with any organizations that might have an interest in the submitted work. Other relationships: All authors have declared that there are no other relationships or activities that could appear to have influenced the submitted work.

\section{References}

1. Gilani GM, Kamal S, Akhter AS: A differential study of breast cancer patients in Punjab, Pakistan. J Pak Med Assoc. 2003, 53:478-481.

2. Bhurgri Y, Bhurgri A, Nishter S, et al.: Pakistan - country profile of cancer and cancer control 1995-2004. J Pak Med Assoc. 2006, 56:124-130.

3. Flobbe K, Bosch AM, Kessels AG, et al.: The additional diagnostic value of ultrasonography in the diagnosis of breast cancer. Arch Intern Med. 2003, 163:1194-1199.

10.1001/archinte.163.10.1194

4. Weir HK, Thun MJ, Hankey BF, et al.: Annual report to the nation on the status of cancer, 1975-2000, featuring the uses of surveillance data for cancer prevention and control. J Natl Cancer Inst. 2003, 3:1276-1299. 10.1093/jnci/djg040

5. Tabar L, Yen MF, Vitak B, Chen HH, Smith RA, Duffy SW: Mammography service screening and mortality in breast cancer patients: 20-year follow-up before and after introduction of screening. Lancet. 2003, 361:1405-1410. 10.1016/S0140-6736(03)13143-1

6. Elmore JG, Armstrong K, Lehman CD, Fletcher SW: Screening for breast cancer. JAMA. 2005, 293:1245-1256. 10.1001/jama.293.10.1245

7. Pisano ED, Gatsonis C, Hendrick E, et al.: Diagnostic performance of digital versus film mammography for breast-cancer screening. N Engl J Med. 2005, 353:1773-1783. 10.1056/NEJMoa052911

8. Pisano ED, Yaffe MJ: Digital mammography. Radiology. 2005, 234:353-361. 10.1148/radiol.2342030897

9. Pisano ED, Cole EB, Major S, Zong S, et al.: Radiologists' preferences for digital mammographic display. Radiology. 2000, 216:820-830. 10.1148/radiology.216.3.r00se48820 
10. Yunus M, Ahmed N, Masroor I, Yaqoob J: Mammographic criteria for determining the diagnostic value of microcalcifications in the detection of early breast cancer. J Pak Med Assoc. 2004, 54:24-29.

11. Pisano ED, Hendrick RE, Yaffe MJ, et al.: Diagnostic accuracy of digital versus film mammography: exploratory analysis of selected population subgroups in DMIST. Radiology. 2008, 246:376-383. 10.1148/radiol.2461070200

12. Lukong KE: Understanding breast cancer - the long and winding road . BBA Clin. 2017, 7:6477. 10.1016/j.bbacli.2017.01.001

13. Iqbal J, Bano K, Saeed A, Akram M, Aziz Z: Survival of women with locally advanced breast cancer at a teaching hospital in Lahore. J Pak Med Assoc. 2010, 60:721-725.

14. Shulman LN, Willett W, Sievers A, Knaul FM: Breast cancer in developing countries: opportunities for improved survival. J Oncol. 2010, 2010:595167. 10.1155/2010/595167

15. Ciatto S, Morrone D, Catarzi S, Bonardi R: Breast cancer reliability of mammographic appearance as a predictor of hormone receptor status. Radiology. 1992, 182:805-808. 10.1148/radiology.182.3.1311118

16. Naeem M, Khan N, Aman Z, Nasir A, Samad A, Khattak A: Pattern of breast cancer: experience at Lady Reading Hospital, Peshawar. J Ayub Med Coll Abbottabad. 2008, 20:22-25.

17. Kettritz U, Morack G, Decker T: Stereotactic vacuum-assisted breast biopsies in 500 women with microcalcifications: radiological and pathological correlations. J Ayub Med Coll Abbottabad. 2005, 55:270-276. 10.1016/j.ejrad.2004.10.014

18. Kaul D, Fallenberg E, Diekmann F, Budach V, Maurer M: Dislocability of localization devices for nonpalpable breast lesions: experimental results. Radiol Res Pract. 2014, 2014:425823. $10.1155 / 2014 / 425823$

19. Chauhan A, Subba SH, Menezes RG, et al.: Younger women are affected by breast cancer in South India - a hospital-based descriptive study. Asian Pac J Cancer Prev. 2011, 12:709-711.

20. Mai PL, Garceau AO, Graubard BI, et al.: Confirmation of family cancer history reported in a population-based survey. J Natl Cancer Inst. 2011, 18:788-797. 10.1093/jnci/djr114

21. Czernichow C, This P, Asselain B, et al.: HRT in post menopausal women and breast cancer at the Institut Curie. Bull Cancer. 2007, 94:469-475.

22. Akhtar W, Aslam M, Ali A, Mirza K, Ahmad N: Film retakes in digital and conventional radiography. J Coll Physicians Surg Pak. 2008, 18:151-153.

23. Dershaw DD: Status of mammography after the digital mammography imaging screening trial digital versus film. Breast J. 2006, 12:99-102. 10.1111/j.1075-122X.2006.00216.x 